\title{
Berichtigung zu Dreyer New General Catalogue of Nebulae.
}

Der Ort des in dem Catalog unter Nr. 3760 aufgeführten Nebels beruht auf einer Beobachtung von D'Arrest I863 Febr. 2 r. D'Arrest's Beschreibung lautet: Parvula, clara; in centro multo lucidior $=* 13$ magn. . * Jo-1 I magn. praec, 4.० I 75" ad austrum. Im Januar 1865 suchte D'Arrest den Nebel vergeblich an dem angegebenen Orte und auch mit Lord Rosse's Instrumenten konnte der Nebel an vier Beobachtungstagen nicht attgefunden werden. Statt seiner aber fand Copeland in der Nähe des D'Arrest'schen Ortes eine Gruppe von 7 Nebeln. Als ich vor kurzem mit dem 18 z. Refractor den Nebel aufsuchen wollte, fand ich ihn ebenfalls nicht, aber auch nicht die Copeland'sche Nebelgruppe. Ich habe nun die folgende Erklärung dieses Um standes gefunden.

D'Arrest's Beobachtung ist in AR. mit einem Fehler von $\mathbf{I}^{\text {h }}$ behaftet. Vermindert man die AR. um diesen Betrag, so wird man ganz genau auf den Ort von NGC. $3301=$ H. II. $4^{6}$ geführt. D'Arrest beschreibt diesen Nebel wie folgt: Clara, minuta, rotunda. Ejus nucleus $=$ I I-I 2 magn.. * Io-I r magn. praec. $3^{5} \cdot 7 \quad 3^{\prime}$ fere ad septentrionem. Die Uebereinstimmung ist bis auf das Zeichen der Declinationsdifferenz eine vollständige. Nach meinen Messungen von NGC. $330 \mathrm{I}$ ist die Position des Sterns gegen den Nebel $\boldsymbol{A}=\mathrm{r} 75^{\prime \prime} \mathrm{I} p=34^{\circ}{ }^{\circ} 0^{\prime}$, so dass also D'Arrest's Beschreibung hier richtig ist und ein Versehen bei der Beobachtung 1863 Febr. 2 I anzunehmen wäre. Ein Zweifel bezüglich der Identificirung dürfte hierdurch wohl nicht entstehen; vielleicht liesse sich durch Einsicht des Beobachtungsjournals die Richtigkeit der Annahme auch beweisen.

Die Copeland'sche Nebelgruppe fand ich nach einigem Suchen in einem Abstande von etwa $25^{\circ}$ von dem in NGC. angegebenen Orte. Dass ein Fehler in der Ortsbestimmung vorliegen müsse, schien schon aus den Aufzeichnungen in

Strassburg I 894 April. den »Observations of nebulae etc. at Birr Castle \& selbst hervorzugehen. Die betreffende Stelle am Himmel ist an vier Abenden beobachtet. Ausser der Copeland'schen Gruppe von 7 Nebeln befinden sich dort noch die etwa gleich hellen Nebel NGC. 3743,3758 , die ebenfalls von Copeland aufgefunden wurden, und von denen wenigstens der letztere mit der Gruppe zugleich im Gesichtsfelde sein müsste, wenn die Dreyer'sche Ortsangabe richtig wäre. In der That kommen aber an zwei Beobachtungstagen nur die beiden zuletzt erwähnten Nebel, an den beiden anderen nur die Nebelgruppe vor. Die Position der Nebel ist bestimmt durch Vergleichung mit einem Stern $10^{\mathrm{m}}-\mathrm{II}^{\mathrm{m}}$, welcher wieder an einen Stern $8^{\mathrm{m}}$ angeschlossen wurde. Dieser Stern $8^{\mathrm{m}}$ soll nach Dreyer's Angabe $=$ BD. $+22^{\circ} .2380$ sein. Copeland nennt ihn einmal very red, das zweite Mal reddish. In der That zeigt jener Stern aber gar keine besondere Färbung. Die Gruppirung der von mir beobachteten Nebelgruppe gegen einen Stern $10^{\mathrm{m}}-\mathrm{I}^{\mathrm{m}}$ stimmt genau überein mit den Copeland'schen Messungen und dieser Stern hat auch die von Copeland geforderte Lage gegen den Stern $7^{\mathrm{m}} \cdot 3$ BD. $+22^{\circ} 23^{85}$; schliesslich ist, um jeden $Z_{w e i f e l}$ zu beseitigen, dieser Stern auch stark röthlich gefärbt.

Es ist demnach in NGC. die Nr. 3760 zu streichen und an die Positionen der Nebel NGC. 3745, 3746, 3748, $375^{\circ}, 375 \mathrm{r}, 3753,3754$ die Correctur anzubringen $\Delta \alpha=$ $+\mathrm{r}^{\mathrm{m}} 3^{\mathrm{s}}, \Delta \delta=+\mathrm{I} 5.9$. Ausserdem ist in den Verzeichnissen rother bez. farbiger Sterne von Birmingham und Fr. Krüger der Stern.BD. +2 2.2380 zu ersetzen durch BD. $+22^{\circ} .23^{8} 5$.

Beim Suchen nach dem D'Arrest'schen Nebel fand ich noch folgende Nova

1860.0

$$
a=\text { I I }^{\mathrm{h}} 26^{\mathrm{m}} 25^{\mathrm{s}} \quad \delta=+22^{\circ} 9^{\prime 2} \quad \text { pF; pS; bM. }
$$

\section{Ist man berechtigt, die Methode der Breitenbestimmung aus reciproken Höhen auf Römer zurückzuführen?}

\section{Von P. Kempf.}

Das Verdienst, die Methode der Polhöhenbestimmung aus reciproken Höhen (gewöhnlich die Talcott'sche genannt) erdacht zu haben, wird in neuester Zeit vielfach für O. Römer in Anspruch genommen. Dem gegenüber möchte ich auf einige Stellen in Peter Horrebow's Schriften hinweisen, aus denen mir mit Sicherheit hervorzugehen scheint, dass dieses Verfahren seine Entstehung nicht Römer verdankt, sondern erst dessen Schüler und Nachfolger Horrebow.

Im Cap. VII des „Atrium Astronomiae" (erschienen 1 732) spricht Horrebow über die Bestimmung der Polhöhe seines Beobachtungsthurmes und giebt dabei in den $\$ 100$ und ff. eine Beschreibung der neuen Beobachtungsmethode, von welcher ich hier die wichtigsten Sätze in möglichst wortgetreuer Uebersetzung wiedergeben möchte: »(\$ IOO) Ich theile hier eine neue Methode mit, die Polhöhe aus reciproken Höhen $z u$ bestimmen, und empfehle dieselbe zum allgemeinen Gebrauche, da sie auf einem Observatorium, wenn die Declinationen der Fixsterne und der Sonne in den Solstitien durch das Instrument des Observatoriums oder anderswoher gegeben sind, sehr bequem zum Gebrauche sein kann. ...(\$ IOI) Man nehme ein dreifüsiges Rohr mit guten Gläsern und starkem Oculare und in Brennpunkte mit mehreren parallelen und aequidistanten Fäden versehen, welche in der Mitte von einem senkrechten Faden geschnitten werden. Aus dem Laufe eines Fixsterns in der Nähe des Aequators oder anderswie bestimme man die Entfernung der beiden äussersten Parallelfäden und damit durch Division die einzelnen Intervalle. ...(\$ ro2) Diesen Tubus befestige man an der Speiche eines Rades (asseri rotae) oder einem sonstigen nahe ebenen Gegenstand und hänge dieses Instrument so auf, dass das Netz des Fernrohrs nach Norden einen Stern von bekannter Declination fasst, und nach 